\title{
Recent KTH Contributions to the Understanding of Wall Turbulence
}

\author{
Ramis Örlü and Philipp Schlatter \\ Linné FLOW Centre, KTH Mechanics, Stockholm, Sweden \\ ramis@mech.kth.se \& pschlatt@mech.kth.se
}

\begin{abstract}
The present paper serves as an accompanying document to the two keynote lectures of the present authors at the 4th International Conference on Jets, Wakes and Separated Flows (4th ICJWSF) held at Nagoya, Aichi, Japan from September 17th to 21st, 2013. Namely "Turbulent boundary layers in pipes and on plates: Tripping, suction, structures, and pressure gradients" by Philipp Schlatter and "High Reynolds number scaling in wallbounded flows: A Stockholm perspective" by Ramis Örlü. In particular it is intended as a summary of the authors own publications.
\end{abstract}

\section{EARLY WORK}

The logarithmic velocity distribution as well as the logarithmic friction law are both the outcome of research carried out in the 1920 s and 30 s, where the two leading scientists were Theodore von Kármán and Ludwig Prandtl, sometimes collaborating, sometimes strongly competing. The two cornerstones of wall turbulence were presented by von Kármán at the Third International Congress for Applied Mechanics in Stockholm (25th August 1930). This presentation is usually seen as the birth (or at least 'baptizing') of the logarithmic law (for more details see Ref. [1]).

The study of wall turbulence has a long tradition at KTH Mechanics with some early work dating back to the 60ies [2], while more prominent contributions are those of Alfredsson and Johansson [3, 4, 5, 6] from the 80ies. In particular the experiments by Österlund [7] on high Reynolds number $(R e)$ zero-pressure gradient (ZPG) turbulent boundary layer (TBL) and the direct numerical simulation (DNS) study by Skote [8] on ZPG and adverse pressure gradient (APG) TBL flows still serve as well-documented and well-performed experimental and numerical data sets for the wall turbulence community.

\section{ZPG TBL FLOWS}

The recent joint experimental and numerical efforts at KTH Mechanics were initiated with the work by Schlatter et al. [9] of a spatially developing ZPG TBL flow up to a Reynolds number of $R e_{\theta}=2500$, based on momentum thickness and free-stream velocity. For the first time direct comparisons of DNS and experiments of turbulent boundary layers at the same computationally high and experimentally low $R e_{\theta}$ were given, showing excellent agreement in skin friction, mean velocity, and turbulent fluctuations. These results allow for a substantial reduction of the uncertainty of boundary-layer data, and cross validate the numerical setup and experimental technique.

These efforts were then extended to $R e_{\theta}=4300$ by means of large eddy simulations (LES) [10] upon which a DNS on a finer grid was performed reaching the same $R e$ [11]. The resolution is comparable to high- $R e$ channel-flow simulations such as e.g. Hoyas \& Jiménez [12] and slightly higher than in the previous boundarylayer simulation [9]. The same publication also presents a unique compilation of seven different DNSs pertaining to a canonical TBL under ZPG; such an assessment has previously only been performed on experimental data sets (see e.g. Refs. [13, 14]). Although all the simulations relate to the same physical flow case, the approaches differ in the applied numerical method, grid resolution and distribution, inflow generation method, boundary conditions and box dimensions. The resulting comparison shows surprisingly large differences not only in both basic integral quantities such as the friction coefficient (see Fig. 1a) or the shape factor, but also in their predictions of mean and fluctuation profiles far into the sublayer. It is thus shown that the numerical simulation of TBLs is, mainly due to the spatial development of the flow, very sensitive to, e.g. proper inflow condition, sufficient settling length and appropriate box dimensions. Thus, a DNS has to be considered as a numerical experiment and should be the subject of the same scrutiny as experimental data. However, if a DNS is set up with the necessary care, it can provide a faithful tool to predict even such notoriously difficult flow cases with great accuracy.

In order to trace back the reason for the unexpectedly large differences between various DNS data sets, the effect of inflow and tripping effects in ZPG TBL flows was systematically investigated by means of DNS [15]; see also the featuring article by Hutchins [17]. The downstream evolution of integral quantities (see Fig. 1b) as well as mean and fluctuation profiles is analysed, and the results show that different inflow conditions and tripping effects do indeed explain most of the differences observed when comparing available DNS at low Re. It 

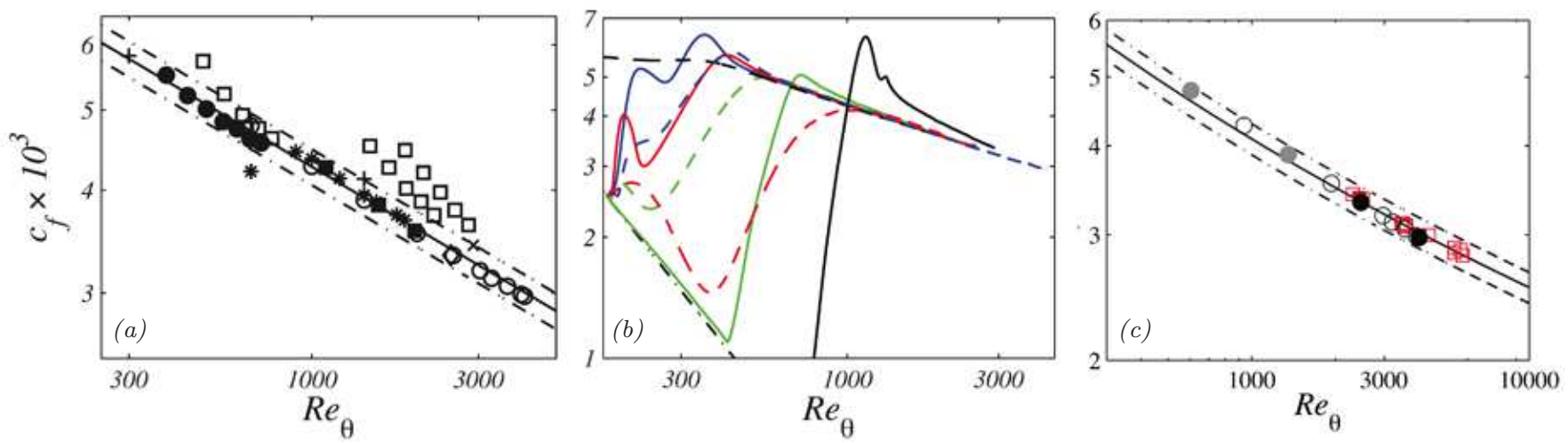

Fig. 1 Skin friction factor $\left(c_{f}\right)$ as function of Reynolds number $\left(R e_{\theta}\right)$ for flat plate turbulent boundary layer flows. (a) Compilation of 8 independent well-resolved DNS from literature [11]. (b) DNS with different inflow length and tripping effects [15]. (c) One-to-one comparison between in-house DNS $(\bigcirc)$ and experiment ( $\square)$ [16].
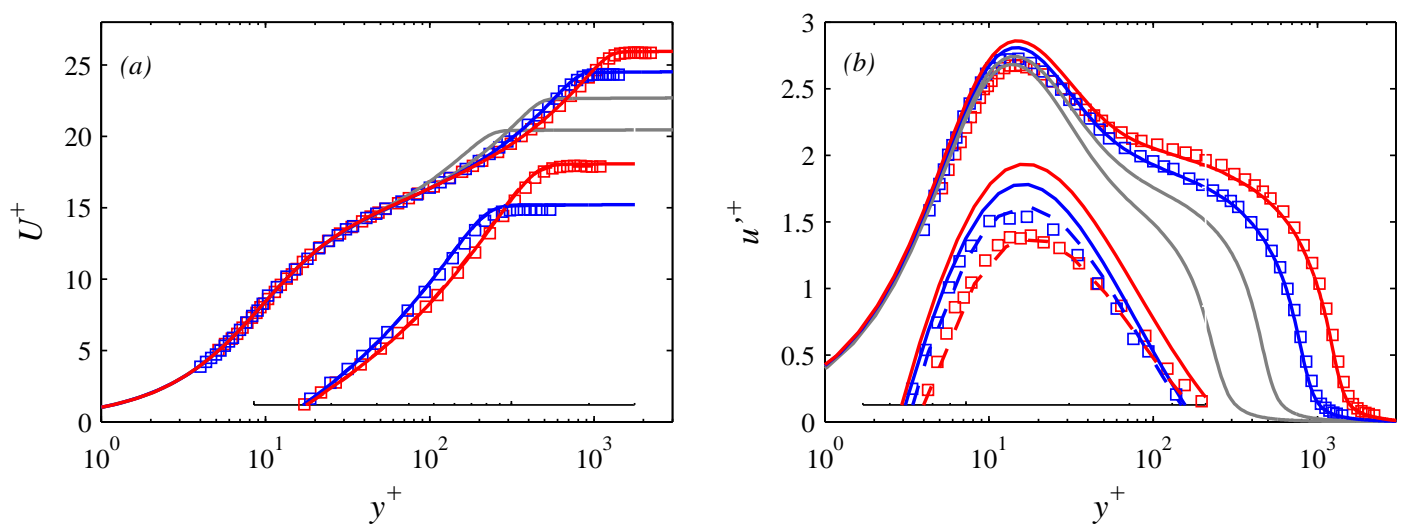

Fig. 2 Inner-scaled (a) mean streamwise velocity and (b) streamwise root mean square profile: numerical (-) and experimental data $(\square)$. Insert enlarges the near-wall peak region, in which profiles from spanwise averaged DNS data with equivalent experimentally $L^{+}$values are given $(---)$as well.

is further found that, if transition is initiated inside the boundary layer at a low enough Reynolds number $R e_{\theta}<300$, all quantities agree well for both inner and outer layer for $R e_{\theta}>2000$. This result gives a lower limit for meaningful comparisons between numerical and/or wind tunnel experiments, assuming that the flow was not severely over- or understimulated. It is further shown that even profiles of the wall-normal velocity fluctuations and Reynolds shear stress collapse for higher $R e_{\theta}$ irrespective of the upstream conditions. In addition, the overshoot in the total shear stress within the sublayer observed in the DNS of Wu \& Moin [18] has been identified as a feature of transitional boundary layers.

Based on these results, a detailed comparison between the aforementioned DNS and experiment [16] of a turbulent boundary layer under ZPG conditions at moderate Reynolds numbers with an overlapping range of $2300<R e_{\theta}<4300$ has been performed [19, 20]. Integral and global quantities have been found to agree very well and confirm quantitatively the correlation by Monkewitz et al. [21] for the shape factor and skinfriction coefficient (see Fig. 1c). Mean and fluctuating streamwise velocity profiles (as shown in Fig. 2), includ- ing higher-order moments, and the probability density distribution (see Fig. 4) have been found to agree remarkably well throughout the boundary layer. Differences within the buffer region for the higher order moments could solely be related and traced back to insufficient spatial resolution effects of the employed hot-wire sensor [22], thereby highlighting the statistical identity of both the present experimental and numerical data sets. Similarly, structural quantities, like the large scale transport of small scale energy related to the amplitude modulation of the small scales by means of large-scale fluctuations [23], have been shown to be alike to a high degree. The established identity of both data sets persists as well for the energy spectra. In particular, the spanwise averaged DNS matched with the viscous-scaled wire length is found to agree throughout the boundary layer for the complete range of temporal frequencies at $R e_{\theta}=2500$. For the comparison at $R e_{\theta}=4000$, on the other hand, the experiment exhibits a (weak) secondary peak in the outer region, related to structures of length 5-7 boundary-layer thicknesses. In the DNS, this outer peak is less clear and slightly moved to shorter temporal periods. The discrepancy is thought to be related to the 
restricted domain width in the DNS, which in turn hinders the very large scale motions to grow. In this respect ongoing simulations of a turbulent asymptotic suction boundary layer (ASBL) might give some answers, where artificially using smaller domains, i.e. limiting the size of the largest turbulent structures, gave unexpected results [24] even in the mean profiles.

The aforementioned efforts have recently also been extended to a new highly resolved large-eddy simulation was presented for a spatially developing turbulent boundary layer, covering in a single domain the range $R e_{\theta}=180$ to 8300 . Turbulence statistics and integral values are in close agreement with experiments and other simulations. The evolution of the large outer-layer structures was examined using spectra and a $k_{z}^{-1}$ range was observed for the streamwise velocity $[25,26]$. These data sets are currently visualized and post-processed in order to extract the dominant flow structures in the turbulent near-wall region. In particular, the question of whether distinct hairpin vortices are present close to the wall is addressed. The present results clearly suggest that in turbulent boundary layers, hairpin vortices may exist at low Reynolds numbers, induced by laminar-turbulent transition. However, they do not persist in great number the fully developed region. Their dominant appearance as instantaneous coherent structures in the outer boundary-layer region is very unlikely [27, 28].

\section{SPATIAL RESOLUTION: ASSESSMENT \& CORRECTION}

Coming back to the near-wall peak in the root mean square (rms) profiles, the DNS data can be made to match nicely the experimental profile when the timeseries is averaged in the spanwise direction before computing the statistics (see e.g. Ref. [29]) as demonstrated in the insert of Fig. 2. While there exist a number of heuristic models to correct rms profiles (e.g. Ref. [30, 31]), these need to be calibrated against available data. Following the work by Segalini et al. [32] we have shown [33] that by using two single hot-wire sensors of different lengths $L$, it is possible from measurements of the variance of the streamwise turbulence fluctuations to correct for the effect of spatial averaging along the sensors. In addition to the correction of the level of the variance it is also possible to obtain an estimate of the transverse Taylor microscale $\lambda_{g}$ from the same data set. Fig. 3 shows the results obtained using the proposed method. The profiles have been reconstructed across the full boundary layer height using pairing of different wire lengths. The overall collapse of the reconstructed signals with the original data is very good at all distances from the wall as well as for a large variety of $L^{+}$combinations.

The theoretical model, introduced in Segalini et al. [32] quantifying the effect of a linear spatial filter of hotwire probes on the mean and the variance of the streamwise velocity in turbulent wall-bounded flows, has also been extended to describe the effect of spatial filtering

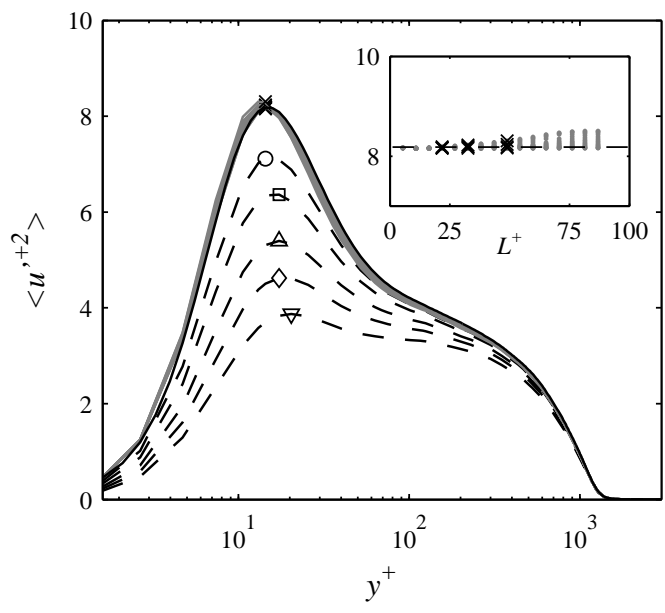

Fig. 3 Inner-scaled variance profiles from a DNS [11] of a ZPG TBL flow at $R e_{\tau} \approx 1300$ : black solid line. Profiles with progressively larger $L^{+}$values, i.e. $L^{+}$of 22,33 , 49, 65 and 87, are simulated by spanwise filtering of the DNS: dashed lines. Variance profiles extracted through the method proposed in Ref. [33].

on the third and fourth-order moments of the same velocity component $[34,35]$.

Once the effect of spatial resolution on higher order moments is assessed Reynolds number trends start to become unambiguous. In particular discussions regarding the near-wall peak $[36,37]$, the so called "outer" peak $[38,39]$, or the fluctuating wall shear stress [40] could to a large extend be resolved. Parallel to these efforts, the DNS data has also been exploited to assess common correction methods for Pitot tube measurements in wall-bounded turbulent flows [41] as well as the effect of temperature gradients on hot-wire measurements [42], or extend scaling laws for pressure fluctuations [43].

\section{VISCOUS SUBLAYER}

The aforementioned comparison between DNS and experimental data has pointed out that DNS resembles the experimental data in integral, mean and higher order quantities as well as spectral quantities when spatial resolution effects are taken into account to a high degree as demonstrated through the contour map of the probability density functions (PDF) depicted in Fig. 4 [44]. The advantage of having access to intrinsic details of the DNS and experimental data, and a consistent reevaluation of integral and statistical quantities highlights every apparent difference between the data sets as something worth to investigate. Such an apparent difference appears e.g. in the viscous sublayer, a region difficult to explore experimentally. Hot-wire measurements are known to be affected by near-wall effects in the viscous sublayer which lead the measured mean velocity to appear higher than it actually is. The detailed comparison of the PDF in Fig. 4 shows that the region in which the hot-wire experiences additional heat losses to the wall is not only a function of the wall distance, but dependent 

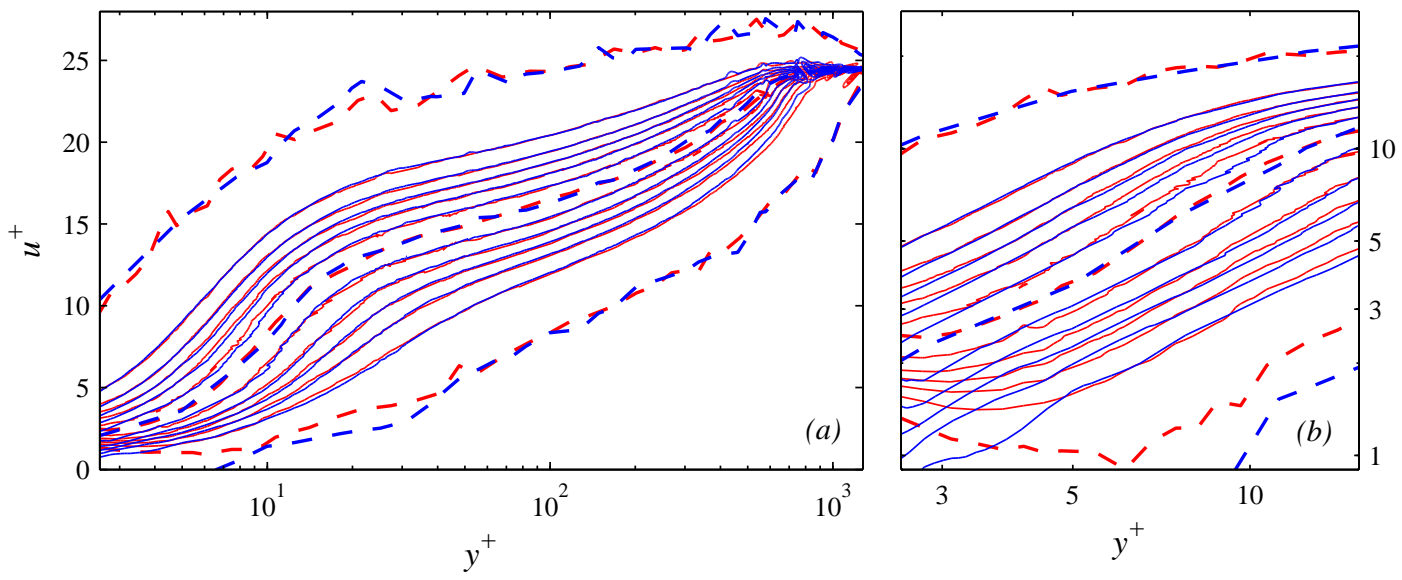

Fig. 4 Probability density distribution map of the streamwise velocity at $R e_{\theta}=2500$ for experimental (red) and DNS (blue) data. Solid contours indicate 10,30, 50, 70, and $90 \%$ of the local peak PDF value (centered dashed line), whereas the outer dashed lines include all sampled velocity signals, i.e. the extreme values of the PDF.

on the instantaneous velocity as well. Since the high velocity fluctuations within the viscous sublayer follow the DNS (parallel contours) this part of the PDF can actually be exploited to obtain an accurate estimate of the wall position and friction velocity [45].

Interesting with respect to the viscous sublayer is also that a region with clearly negative shear stress (instantaneous flow reversal) exists [40]. The occurrence is seldom (below $0.1 \%$ ) but not negligible. These rare negative streamwise velocities and extreme wall-normal velocity fluctuations near the wall are investigated for turbulent channel flow at a series of Reynolds numbers based on friction velocity up to $R e_{\tau}=1000$. A strong oblique vortex outside the viscous sublayer was found to cause this backflow [46]. Similarly, the very large values of the normalized fourth moment (flatness) of the wallnormal velocity could also be confirmed to be of physical nature, caused by a pair of counter-rotating vortices in the immediate near-wall region.

\section{FROM STRAIGHT TO BEND PIPES}

While the focus of recent work was clearly on ZPG TBL flows, efforts are now shifted towards more complex canonical flow cases. In particular large-scale fully resolved DNSs of incompressible pipe flows in long computational domains have been performed at $R e_{\tau}=$ $180,360,550$ and 1000 [47]. The new data is put into perspective with other simulation data sets, obtained in pipe, channel and boundary layer geometry as well as compared with in-house experimental data [48, 37].

These data also serve as a baseline for DNSs in curved pipes at moderate Reynolds numbers. After the validation of data and setup against existing DNS results, a comparative study of turbulent characteristics at different bulk Reynolds numbers $R e_{b}=5300$ and 11700, and various curvature parameters $\kappa=0,0.01,0.1$ is presented [49]. Ongoing experiments by means of high speed particle image velocimetry in pipe bends are performed in close collaboration $[50,51]$.

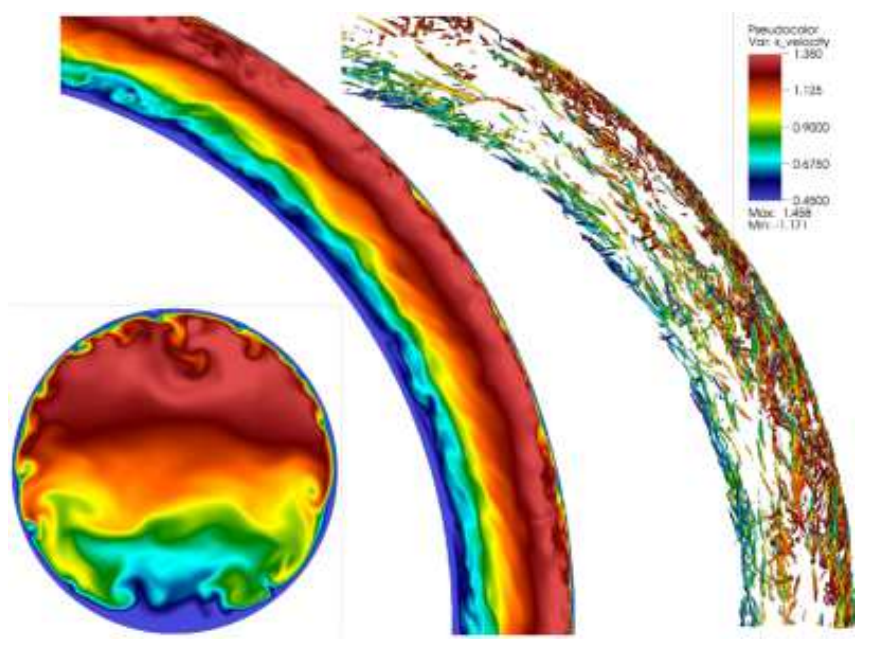

Fig. $53 \mathrm{D}$ rendering of isosurfaces of negative $\lambda_{2}$ coloured by the velocity magnitude, equatorial mid-plane view of streamwise velocity, and cross-sectional view of the same quantity of the turbulent flow at strongly curved pipe $(\kappa=0.1)$ with and $R e_{b}=11700$ [52].

\section{REFERENCES}

[1] PH Alfredsson, S Imayama, RJ Lingwood, R Örlü, and A Segalini. Turbulent boundary layers over flat plates and rotating disks-the legacy of von Kármán: a Stockholm perspective. Eur. J. Mech. B-Fluid, 40:17-29, 2013.

[2] ER Lindgren. The transition process and other phenomena in viscous flow. Arkiv Fysik, 1957.

[3] AV Johansson and PH Alfredsson. On the structure of turbulent channel flow. J. Fluid Mech., 122:295314, 1982. 
[4] AV Johansson and PH Alfredsson. Effects of imperfect spatial resolution on measurements of wallbounded turbulent shear flows. J. Fluid Mech., 137:409-421, 1983.

[5] PH Alfredsson and AV Johansson. On the detection of turbulence-generating events. J. Fluid Mech., 139:325-345, 1984.

[6] PH Alfredsson, AV Johansson, JH Haritonidis, and $\mathrm{H}$ Eckelmann. The fluctuating wall-shear stress and the velocity field in the viscous sublayer. Phys. Fluids, 31:1026-1033, 1988.

[7] JM Österlund. Experimental Studies of Zero Pressure-gradient Turbulent Boundary Layer Flow. Ph.D. thesis, Royal Institute of Technology, Stockholm, Sweden, 1999

[8] M Skote. Studies of turbulent boundary layer flow through direct numerical simulation. Ph.D. thesis, Royal Institute of Technology, Stockholm, Sweden, 2001.

[9] P Schlatter, R Örlü, Q Li, G. Brethouwer, JHM Fransson, AV Johansson, PH Alfredsson, and DS Henningson. Turbulent boundary layers up to $R e_{\theta}=2500$ studied through simulation and experiment. Phys. Fluids, 21:051702, 2009.

[10] P Schlatter, Q Li, G Brethouwer, AV Johansson, and DS Henningson. Simulations of spatially evolving turbulent boundary layers up to $R e_{\theta}=4300$. Int. J. Heat Fluid Flow, 31:251-261, 2010.

[11] P Schlatter and R Örlü. Assessment of direct numerical simulation data of turbulent boundary layers. $J$. Fluid Mech., 659:116-126, 2010.

[12] S Hoyas and J Jiménez. Scaling of the velocity fluctuations in turbulent channels up to $R e_{\tau}=2003$. Phys. Fluids, 18:011702, 2006.

[13] HH Fernholz and PJ Finley. The incompressible zero-pressure-gradient turbulent boundary layer: An assessment of the data. Prog. Aerosp. Sci., $32: 245-311,1996$

[14] KA Chauhan, PA Monkewitz, and HM Nagib. Criteria for assessing experiments in zero pressure gradient boundary layers. Fluid Dyn. Res., 41:021404, 2009 .

[15] P Schlatter and R Örlü. Turbulent boundary layers at moderate Reynolds numbers: inflow length and tripping effects. J. Fluid Mech., 710:5-34, 2012.

[16] R Örlü. Experimental studies in jet flows and zero pressure-gradient turbulent boundary layers. Ph.D. thesis, Royal Institute of Technology, Stockholm, Sweden, 2009.

[17] N Hutchins. Caution: tripping hazards. J. Fluid Mech., 710:1-4, 2012.
[18] $\mathrm{X} \mathrm{Wu}$ and $\mathrm{P}$ Moin. Transitional and turbulent boundary layer with heat transfer. Phys. Fluids, 22:085105, 2010.

[19] R Örlü and P Schlatter. Turbulent boundary-layer flow: comparing experiments with DNS. Progress in Turbulence and Wind Energy IV, Proc. iTi Conf. Turbulence, Bertinoro, Italy, 141:213-216, 2012.

[20] R Örlü and P Schlatter. Comparison of experiments and simulations for zero-pressure gradient turbulent boundary layers at moderate Reynolds numbers. Exp. Fluids, 54:1547, 2013.

[21] PA Monkewitz, KA Chauhan, and HM Nagib. Comparison of mean flow similarity laws in zero pressure gradient turbulent boundary layers. Phys. Fluids, 20:105102, 2008

[22] R Örlü and PH Alfredsson. On spatial resolution issues related to time-averaged quantities using hotwire anemometry. Exp. Fluids, 49:101-110, 2010.

[23] P Schlatter and R Örlü. Quantifying the interaction between large and small scales in wall-bounded turbulent flows: A note of caution. Phys. Fluids, 22:051704, 2010 .

[24] P Schlatter and R Örlü. Turbulent asymptotic suction boundary layers studied by simulation. $J$. Phys.: Conf. Ser., 318:022020, 2011.

[25] P Schlatter and Q Li. Turbulent boundary layers in long computational domains. Progress in Turbulence V, Proc. iTi Conf. Turbulence, Bertinoro, Italy (in Press), 2013.

[26] G Eitel-Amor, R Örlü, and P Schlatter. Turbulent boundary layers in long computational domains. $D i$ rect and Large-Eddy Simulation IX, 2013.

[27] P Schlatter, Q Li, G Brethouwer, AV Johansson, and DS Henningson. Structure of a turbulent boundary layer studied by DNS. Direct and LargeEddy Simulation VIII, 15:9-14, 2011.

[28] P Schlatter, R Örlü, G Eitel-Amor, Q Li, DS Henningson, and F Hussain. On the near-wall vortical structures at moderate Reynolds numbers. Proc. MekIT'13, Trondheim, Norway., 2013.

[29] R Örlü, JHM Fransson, and PH Alfredsson. On near wall measurements of wall bounded flows-The necessity of an accurate determination of the wall position . Prog. Aerosp. Sci., 46:353-387, 2010.

[30] PA Monkewitz, RD Duncan, and HM Nagib. Correcting hot-wire measurements of stream-wise turbulence intensity in boundary layers. Phys. Fluids, 22:091701, 2010 . 
[31] AJ Smits, JP Monty, M Hultmark, SCC Bailey, $\mathrm{N}$ Hutchins, and I Marusic. Spatial resolution correction for wall-bounded turbulence measurements. J. Fluid Mech., 676:41-53, 2011.

[32] A Segalini, A Cimarelli, J-D Rüedi, E De Angelis, and A Talamelli. Effect of the spatial filtering and alignment error of hot-wire probes in a wall-bounded turbulent flow. Meas. Sci. Tech., 22:105408, 2011.

[33] A Segalini, R Örlü, P Schlatter, PH Alfredsson, J-D Rüedi, and A Talamelli. A method to estimate turbulence intensity and transverse Taylor microscale in turbulent flows from spatially averaged hot-wire data. Exp. Fluids, 51:693-700, 2011.

[34] A Talamelli, A Segalini, R Örlü, P Schlatter, and $\mathrm{PH}$ Alfredsson. A method to correct third and fourth order moments in turbulent flows. J. Phys.: Conf. Ser., 318:042023, 2011.

[35] A Talamelli, A Segalini, R Örlü, P Schlatter, and $\mathrm{PH}$ Alfredsson. Correcting hot-wire spatial resolution effects in third- and fourth-order velocity moments in wall-bounded turbulence. Exp. Fluids, $54: 1496,2013$

[36] N Hutchins, TB Nickels, I Marusic, and MS Chong. Hot-wire spatial resolution issues in wall-bounded turbulence. J. Fluid Mech., 635:103-136, 2009.

[37] R Örlü and PH Alfredsson. Comment on the scaling of the near-wall streamwise variance peak in turbulent pipe flows. Exp. Fluids, 54:1431, 2012.

[38] PH Alfredsson, A Segalini, and R Örlü. A new scaling for the streamwise turbulence intensity in wallbounded turbulent flows and what it tells us about the "outer" peak. Phys. Fluids, 23:041702, 2011.

[39] PH Alfredsson, R Örlü, and A Segalini. A new formulation for the streamwise turbulence intensity distribution in wall-bounded turbulent flows. Eur. J. Mech. B-Fluid, 36:167-175, 2012.

[40] R Örlü and P Schlatter. On the fluctuating wall shear stress in zero pressure-gradient turbulent boundary layer flows. Phys. Fluids, 23:021704, 2011.

[41] SCC Bailey, M Hultmark, JP Monty, PH Alfredsson, MS Chong, RD Duncan, JHM Fransson, N Hutchins, I Marusic, BJ McKeon, HM Nagib, R Örlü, A Segalini, AJ Smits, and R Vinuesa. Obtaining accurate mean velocity measurements in high Reynolds number turbulent boundary layers using Pitot tubes. J. Fluid Mech., 715:642-670, 2013.

[42] F Malizia, A Cimarelli, E De Angelis, P Schlatter, R Örlü, and A Talamelli. Computational study of temperature gradient effects on hot-wire measurements. Proc. 14th EUROMECH European Turbulence Conference, Lyon, France, 2013.

[43] Y Tsuji, S Imayama, P Schlatter, PH Alfredsson, AV Johansson, I Marusic, N Hutchins, and J Monty. Pressure fluctuation in high-Reynolds-number turbulent boundary layer: results from experiments and DNS. J. Turbulence, 13:N50, 2012.

[44] P Schlatter, R Örlü, Q Li, G Brethouwer, AV Johansson, PH Alfredsson, and DS Henningson. Progress in simulations of turbulent boundary layers. Proc. Yth Intl Symp. on Turbulence and Shear Flow Phenomena, Ontario, Canada, 2011.

[45] PH Alfredsson, R Örlü, and P Schlatter. The viscous sublayer revisited-exploiting self-similarity to determine the wall position and friction velocity. Exp. Fluids, 51:271-280, 2011.

[46] P. Lenaers, Q Li, G Brethouwer, P Schlatter, and $\mathrm{R}$ Örlü. Rare backflow and extreme wall-normal velocity fluctuations in near-wall turbulence. Phys. Fluids, 24:035110, 2012.

[47] GK El Khoury, P Schlatter, A Noorani, PF Fischer, G Brethouwer, and AV Johansson. Direct numerical simulation of turbulent pipe flow at moderately high Reynolds numbers. Flow Turbul. Combust. (in Press), 2013.

[48] SS Sattarzadeh, M Ferro, R Örlü, and PH Alfredsson. Revisiting the near-wall scaling of the streamwise variance in turbulent pipe flows. Progress in Turbulence V, Proc. iTi Conf. Turbulence, Bertinoro, Italy (in Press), 2013.

[49] A Noorani, GK El Khoury, and P Schlatter. Evolution of turbulence characteristics from straight to curved pipes. Int. J. Heat Fluid Flow, 41:16-26, 2013.

[50] A Kalpakli, R Örlü, and PH Alfredsson. Dean vortices in turbulent flows: rocking or rolling? J. Vis., 15:37-38, 2011.

[51] A Kalpakli and R Örlü. Turbulent pipe flow downstream a $90^{\circ}$ pipe bend with and without superimposed swirl. Int. J. Heat Fluid Flow, 41:103-111, 2013.

[52] P Schlatter, A Noorani, A Kalpakli, and R Örlü. Coherent motions in turbulent flows through curved pipes. Proc. 14th EUROMECH European Turbulence Conference, Lyon, France, pages 1-2, 2013. 